\title{
Hamiltonian model for multidimensional epistasis
}

\author{
Seher Özçelik ${ }^{1}$ and Ayşe Erzan ${ }^{1,2}$ \\ 1 Department of Physics, Faculty of Sciences and Letters \\ Istanbul Technical University, Maslak 80626, Istanbul, Turkey and \\ 2 Gürsey Institute, P. O. Box 6, Çengelköy 81220, Istanbul, Turkey
}

(Dated: November 13, 2018)

\begin{abstract}
We propose and solve a Hamiltonian model for multidimensional epistastatic interactions between beneficial mutations. The model is able to give rise either to a phase transition between two equilibrium states, without any coexistence, or exhibits a state where hybrid species can coexist, with gradual passage from one wild type to another. The transition takes place as a function of the tolerance of the environment, which we define as the amount of noise in the system.
\end{abstract}

Keywords: Evolution, phase transitions, bit-string models

\section{INTRODUCTION}

Evolution takes place via natural selection, whereby random mutations which have a salutary effect on the fitness (survival probability and/or reproductive capability) of the individual persist in the population and lead to new variants; other, neutral mutations may simply be carried along since they do not affect the well being of the individual. Deleterious mutations usually affect the organism adversely, and the accumulation of too many will reduce the fitness drastically. Each "species" actually consists of a more or less narrow distribution in the phase space of all possible genetic states, and this distribution may shift, in response to environmental pressure, along definite evolutionary routes. [1]

An interesting problem is to explain the rather fast rates at which populations seem to be able to adapt to changing environments, which suggest that the fitness does not depend in a simple additive way on the effects of each independent mutation, but that there is a nonlinear relationship, or epistasis [2], between the effects of point mutations determining the fitness function. In fact, one may surmise that evolution is not a Markovian game, but that the fitness depends upon the history of the successive mutations, in other words, it is a function of the path taken in genomic space. [3] Thus, for a mutation leading to the development of fingers to be beneficial, say, one must already have had a mutation leading to the formation of limbs.

Posed in this way, this problem seems to demand an analysis that is intrinsically dynamical. Yet, it actually lends itself to a treatment in terms of statistical equilibria, with the appropriate choice of a fitness function. In this paper we aim to provide such a fitness function, and solve the resulting model for possibly coexisting equilibrium phases indicating different species.

\section{THE MODEL}

Since Eigen first introduced the quasi-species model [4] there has been a huge amount of both analytical and numerical work on bitstring models of genetic evolu- tion [5, 6], where the genotype of an individual is represented by a string of Boolean variables $\sigma_{i}, i=1, \ldots N$. If one takes the wild type, or the initial genotype, to consist of a string of 0's, each point mutation is indicated by flipping the bit respresenting a given gene, from 0 to 1 . The number of mutations $m$ is then the number of 1's on the whole string, i.e., $m=\sum_{i} \sigma_{i}$. The fitness is usually taken simply to be a function, albeit nonlinear, of $m$.

Clearly, each $i$ 'th variable can be considered as an independent direction in phase space, so that evolution takes place in an $N$ dimensional space, where $N$ is the length of the genome. The genotype is a vertex on an $N$-dimensional unit hypercube, and if only single flips from 0 to 1 are allowed at a time, the path of evolution is a random walk on the edges of this hypercube. One possible way in which a vector variable can be introduced is to consider the whole vector $\mathbf{V} \equiv\left\{\sigma_{i}\right\}$ as the argument of the fitness function. Since the position of each gene on this particular string can be assigned with some arbitrariness, one may then demand that the fitness is only increased relative to the wild type if the bits that flip to 1 occur sequentially. [3]. Thus, $(0,0, \ldots)$, $(1,0, \ldots)(1,1,0 \ldots)$ are in increasing order of fitness while $(0,1, \ldots)$ is less fit.

This demands that we introduce a cost function $H$ which depends on the state $\mathbf{V}$, and we have chosen the fitness $f$ to decrease exponentially with this cost function, viz.,

$$
f \propto e^{-\beta H}
$$

where $\beta$ is a measure of how effective the cost function is, in affecting the fitness. The fitness function $f$ can be identified as the Boltzmann factor in an equilibrium statistical model with the Hamiltonian $H$, at constant inverse "temperature" $\beta^{-1}$. Temperature may be seen as the amount of randomness, or disorder in the system, competing with the cost function in determining the fitness. The higher the temperature, or randomness, the weaker will be the effect of the cost function in determining the state of the system. Therefore we define

$$
T \equiv \beta^{-1}
$$

as the tolerance in the system. 
For the cost function we will borrow a Hamiltonian introduced by Bakk at al. [7], in the context of protein folding, where it is of importance that the folding events take place in a prescribed order. Thus,

$$
H=-\lambda J \sum_{m=1}^{N} \prod_{i=1}^{m} \sigma_{i}-(1-\lambda) J \prod_{i=1}^{N} \sigma_{i}
$$

It can be seen that for $\lambda=0$, the only state which is favorable is that with all $\sigma_{i}=1$, whereas for $0<\lambda \leq 1$, all states with an uninterrupted initial sequence of $1 \mathrm{~s}$ of arbitrary length $m$ lead to improved fitness. Here $J$ is a measure of the strength of the interaction between the states of each of the sites (alleles), $\sigma_{i}$. Clearly, $\beta$ and $J$ will always occur together in this model, in the product $\beta J$, and we may simply absorb $J$ into the definition of $\beta$.

The fitness $f$ may be normalized to take values between $(0,1)$ if we devide the expression in Eq.(11) by the sum over all states $\mathbf{V}$, namely,

$$
Z \equiv \sum_{\left\{\sigma_{i}\right\}} e^{-\beta H\left[\left\{\sigma_{i}\right\}\right]}
$$

This sum may be performed exactly, to give,

$$
Z=\frac{2^{N}-e^{\beta \lambda N}}{2-e^{\beta \lambda}}+e^{[\lambda(N-1)+1] \beta} .
$$

Using this result we may compute the expectation values (average values) of the quantities $\psi_{m} \equiv \prod_{i=1}^{m} \sigma_{i}$, which we shall call, $\Psi_{m}=\left\langle\psi_{m}\right\rangle$, for $m=1, \ldots, N$. Clearly, $\Psi_{m}$ is the probability that in equilibrium, at least $m$ initial loci on the genotype have switched to 1 . One finds,

$$
\Psi_{m}=\frac{1}{Z}\left[\frac{2^{N-m} e^{m \beta \lambda}-e^{N \beta \lambda}}{2-e^{\beta \lambda}}+e^{\beta[(N-1) \lambda+1]}\right] .
$$

Clearly, as $\beta \rightarrow 0, \Psi_{m} \rightarrow(1 / 2)^{m}$, so that it is convenient to define the order parameters

$$
\Phi_{m}=\frac{\Psi_{m}-(1 / 2)^{m}}{1-(1 / 2)^{m}},
$$

which take values in the interval $(0,1)$. In Fig. 1, we present the results of a numerical evaluation of Eq.(6), for $\lambda=0$, and in Fig.2, for $\lambda=1$, as a function of $x \equiv T / J$, which is the ratio of the tolerance in the system to the strength of the epistatic interactions.

We find that for $\lambda=0$, there is a sharp transition for large $N$, at $x>x_{t}$, below which the genotype is completely ordered, with all $\sigma_{i}=1$, while for $x>x_{t}$, the whole population is in the the state with all $\sigma_{i}=0$. From an inspection of Eqs.(5.,7), one sees that $x_{t}=(N \ln 2)^{-1}$. Thus there are only two possible species in this case, with no coexistence between them. However, for $N \rightarrow \infty$, the threshold itself goes to zero. (This can be mended if the strength of the second term in Eq.(3) is chosen to be $N J$ rather than $J$.)

For $\lambda=1$, it can be seen that the sharp phase transition is no longer present (the nonzero value of $\lambda$ has an effect similar to turning on a magnetic field in a magnetic phase transition). For large $x$, all the $\Phi_{m}$ decay exponentially, as $\sim e^{-m / x}$. However, there exist effective thresholds $x_{m}$, for $m>1$, below which there is a nonzero probability of encountering individuals with $m$ initial alleles switched to 1 . This signifies that at any given $x_{m+1}<x<x_{m}$, there is coexistence between $m$ hybrid species, with the first $n \leq m$ alleles in the mutated state. The probability of encountering an individual with $n \leq m$ seqentially mutated alleles is in fact precisely $\Phi_{m}$. We see that $\Phi_{N} \approx 0$ for $x>x_{N}$, with $x_{N} \sim 1 / \ln 2$.

To further elucidate the meaning of "tolerance," we may compute the relative variances $v_{m}$, where

$$
v_{m}^{2} \equiv\left\langle\left(\psi_{m}-\Psi_{m}\right)^{2}\right\rangle / \Psi_{m}^{2} .
$$

It is trivial to note that $\psi_{m}^{2}=\psi_{m}$, so that $v_{m}^{2}=(1-$ $\left.\Psi_{m}\right) / \Psi_{m}$. Then it is straightforward enough to get,

$$
v_{m}^{2}=\frac{1-\left(e^{\beta \lambda} / 2\right)^{m}}{\left(e^{\beta \lambda} / 2\right)^{m}+\left(e^{\beta \lambda} / 2\right)^{N}\left[2 e^{\beta(\lambda-1)}-e^{\beta}-1\right]} .
$$

One may see from here that the behaviour of the system is determined by the critical value of $\beta \lambda$ at $\ln 2$, and moreover, that the variance (or the departure from the ordered phase) also depends on whether $m$ is small or of the order of $N$, as can also be seen clearly from Fig. 2 .

\section{CONCLUSION}

In summary, we have presented a Hamiltonian model of multidimensional epistasis which weights only certain paths in genotype space as being favorable. The model has tunable strength $(J)$ of interactions between different genes, which can be absorbed into an overall parameter $(\beta)$ which determines how tolerant the environment is to deviations from the wildtype, as well as a parameter which decides whether coexistence between hybrid individuals will or will not be allowed. The model exhibits a transition between two pure types as a function of $\beta$ for $\lambda=0$. For $\lambda \neq 0$, low tolerances $T=\beta^{-1}$ give rise to the appearance of hybrid types, in case a given series of mutations increases the fitness.

\section{Acknowledgements}

We are grateful to Güneş Söyler for many useful discussions. AE acknowledge partial support from the Turkish Academy of Sciences.
[1] J. Maynard Smith, Evolutionary Genetics (Oxford University Press, Oxoford, 1998).
[2] A. C. Pai, Foundations of Genetics (McGraw-Hill Inter- 
national Editions), pp.93

[3] F. Kondrashov and A.S. Kondrashov, Proc. Natl. Acad. Sci. USA 98,12089 (2001), and references therein.

[4] M. Eigen, Naturwissenschaften 58, 465 (1971).

[5] D. Stauffer, Comput. Sci. Eng. 1, 78 (1999); D. Stauffer, Physica A 273, 132 (1999); D. Stauffer et al., An. Acad. Bras. Cienc. 73, 15 (2001).

[6] P.M.C. de Oliveira, Computing Boolean Statistical Models (World Scientific, Singapore, 1991).

[7] A. Bakk, J.S. Hoye, A. Hansen, K. Sneppen and G. Zocchi,
Biophysical J. B 6, 157 (1998).

Figure Captions

Fig. 1. The order parameters $\Phi_{m}$ for $\lambda=0$ all differ from zero at the same transition point. Here the length of the genome is 100 . There are no hybrid species.

Fig. 2. The order parameters $\Phi_{m}$ for $\lambda=1$, with $N=100$. There is a set of $N$ effective thresholds, below which hybrid species arise, with $m$ sequentially mutated alleles. 


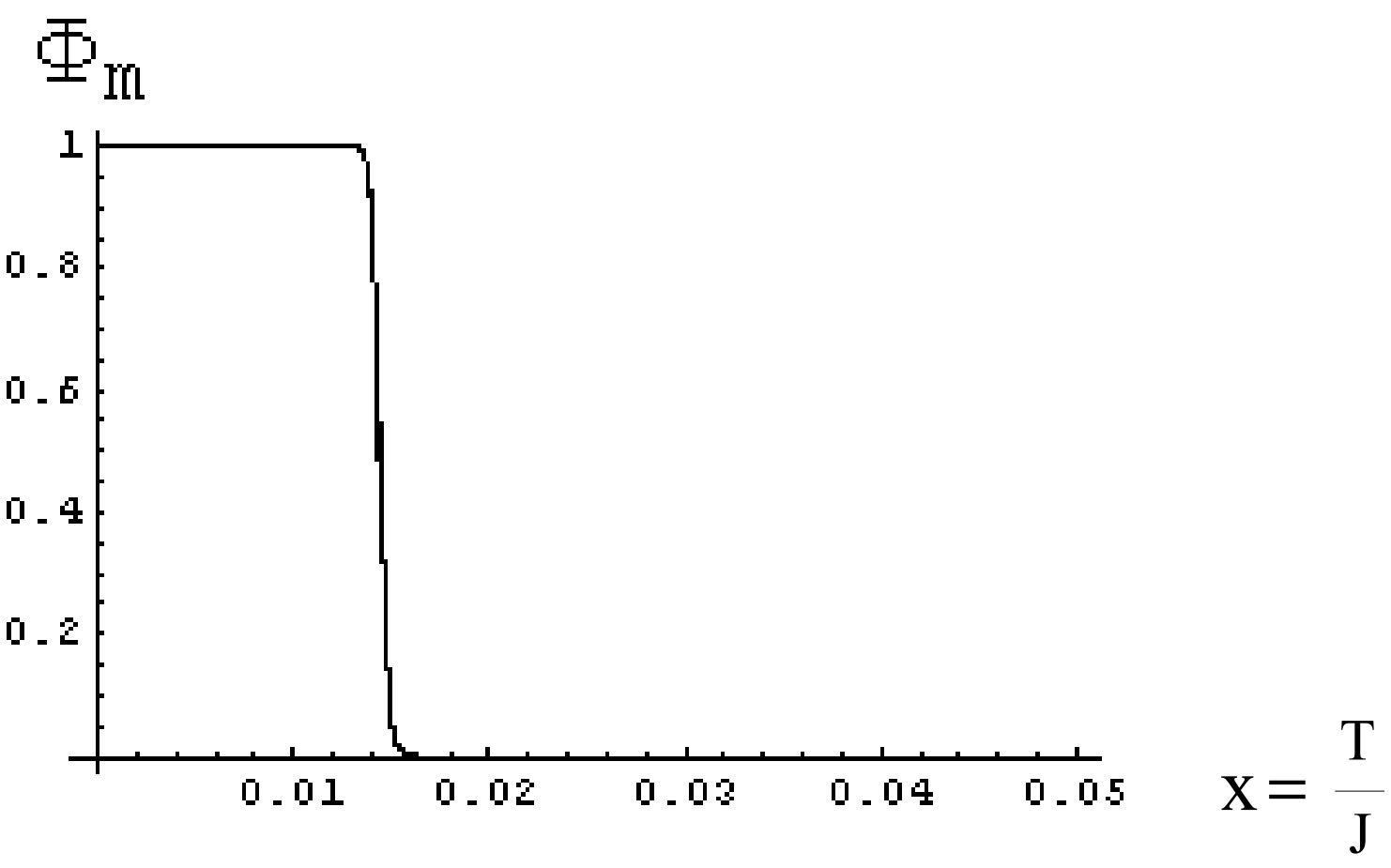




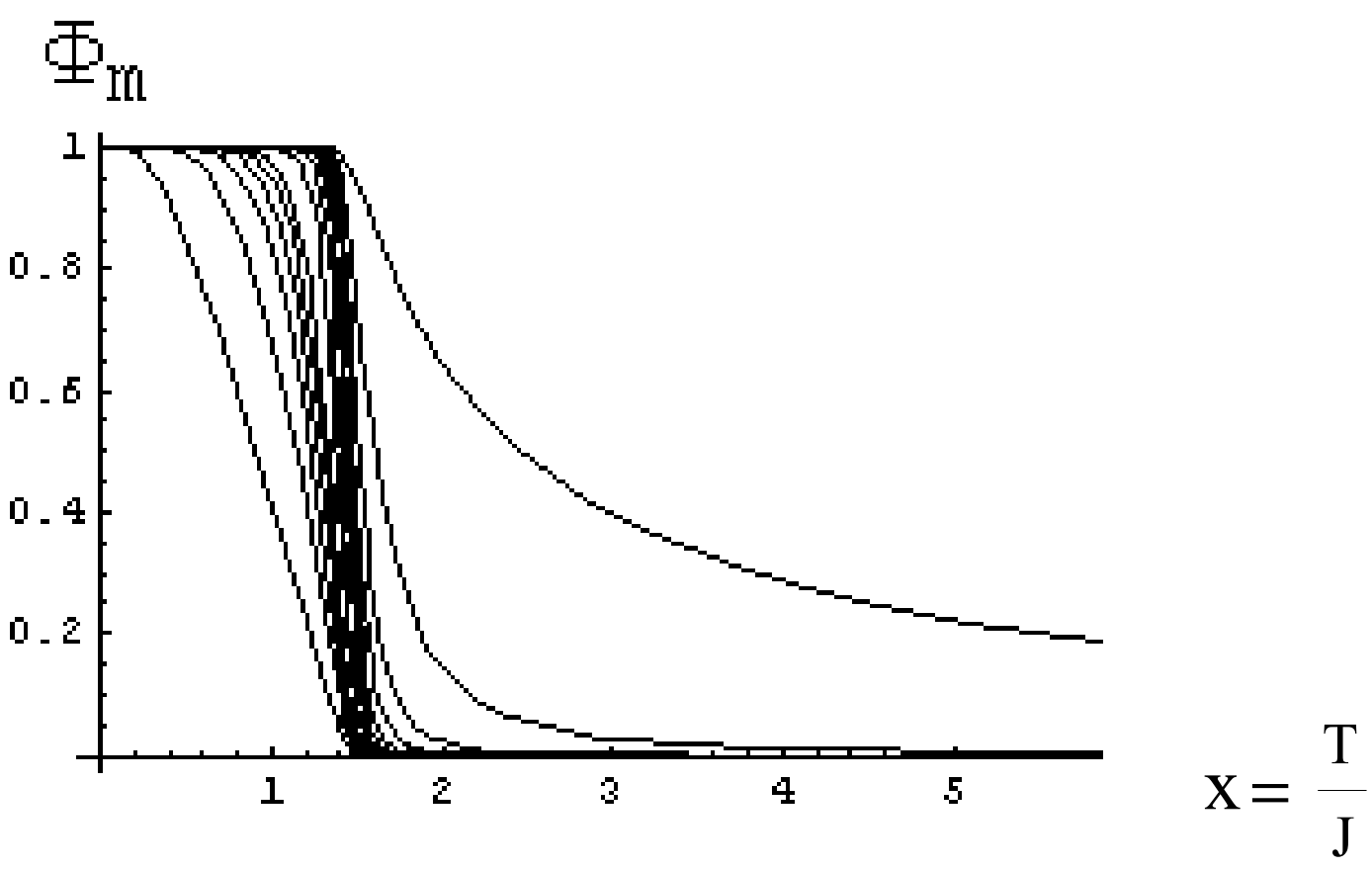

